\title{
A Note on Some Applications of Boyadzhiev's Formula
}

\author{
Gyan Bahadur Thapa ${ }^{1}$, J. López-Bonilla ${ }^{2}$, R. López-Vázquez ${ }^{2}$ \\ ${ }^{1}$ Central Campus, Institute of Engineering, Tribhuvan University, Kathmandu, Nepal, \\ ${ }^{2}$ ESIME-Zacatenco, Instituto Politécnico Nacional, \\ Edif. 4, 1er. Piso, Col. Lindavista CP 07738, CDMX, México \\ Corresponding author: jlopezb@ipn.mx
}

Received: Oct 20, 2017

Revised: Dec 26, 2017

Accepted: Dec 28, 2017

\begin{abstract}
In this paper, we employ an expression of Boyadzhiev to give elementary proofs of the identities for harmonic numbers obtained by PauleSchneider via the Mathematica package Sigma.
\end{abstract}

Keywords: Boyadzhiev's identity, harmonic numbers, Paule-Schneider's relations.

\section{Introduction}

Boyadzhiev $[2,6]$ evaluated several binomial transforms by using Euler's transform for power series and obtained various binomial identities involving power sums with harmonic numbers. Boyadzhiev's Formula for harmonic numbers is as follows:

$\sum_{k=1}^{n}\left(\begin{array}{l}n \\ k\end{array}\right) H_{k} \lambda^{n-k} \mu^{k}=(\lambda+\mu)^{n} H_{n}-\sum_{j=1}^{n} \frac{\lambda^{j}}{j}(\lambda+\mu)^{n-j}$

Paule-Schneider [8] used the Mathematica package Sigma [11] and the Zeilberger's method [9, $13]$ to find the following identities [5] for harmonic numbers [1, 3]:

$$
\begin{gathered}
\sum_{j=1}^{n} H_{j}\left(\begin{array}{l}
n \\
j
\end{array}\right)=2^{n}\left[H_{n}-\sum_{j=1}^{n} \frac{1}{j 2^{j}}\right], \quad H_{n} \equiv \sum_{k=1}^{n} \frac{1}{k}, \\
\sum_{j=1}^{n} j H_{j}\left(\begin{array}{l}
n \\
j
\end{array}\right)=-\frac{1}{2}+2^{n-1}\left[1+n H_{n}-n \sum_{j=1}^{n} \frac{1}{j 2^{j}}\right] .
\end{gathered}
$$

Here we employ (1) to exhibit elementary proofs of these interesting identities.

\section{Paule-Schneider's relations}

In (1) we can use the values $\lambda=\mu=1$ to deduce (2): 
$\sum_{k=1}^{n}\left(\begin{array}{l}n \\ k\end{array}\right) H_{k}=2^{n}\left(H_{n}-\sum_{j=1}^{n} \frac{1}{j 2^{j}}\right) \stackrel{[5]}{=} \sum_{j=1}^{n}(-1)^{j+1}\left(\begin{array}{c}n \\ j\end{array}\right) \frac{2^{n-j}}{j}, \quad n \geq 1$.

The identity (1) with $\lambda=-\mu=1$ implies the relation $[4,10]$ :

$$
\sum_{k=1}^{n}(-1)^{k+1}\left(\begin{array}{l}
n \\
k
\end{array}\right) H_{k}=\frac{1}{n}, \quad n \geq 1,
$$

which is a particular case of the following formula [12] $\left(H_{0}=0\right)$ :

$$
\sum_{k=0}^{n}(-1)^{k+1}\left(\begin{array}{l}
n \\
k
\end{array}\right) H_{k+m}=\frac{1}{n\left(\begin{array}{c}
m+n \\
n
\end{array}\right)}, \quad n \geq 1, \quad m \geq 0 .
$$

Besides, we obtain (6) from [7]:

$$
\sum_{k=0}^{n}(-1)^{k+1}\left(\begin{array}{l}
n \\
k
\end{array}\right) H_{k+s}(x)=\frac{(n-1) !}{(x+s+1)_{n}},
$$

if $x=0$.

Now we apply $\frac{d}{d \mu}$ to (1), thus:

$\sum_{k=1}^{n} k\left(\begin{array}{l}n \\ k\end{array}\right) H_{k} \lambda^{n-k} \mu^{k-1}=n(\lambda+\mu)^{n-1} H_{n}-\sum_{j=1}^{n-1} \frac{n-j}{j} \lambda^{j}(\lambda+\mu)^{n-1-j}$,

which for $\lambda=\mu=1$ gives:

$\sum_{k=1}^{n} k\left(\begin{array}{l}n \\ k\end{array}\right) H_{k}=2^{n-1}\left(n H_{n}-\sum_{j=1}^{n} \frac{n-j}{j 2^{j}}\right)=n 2^{n-1}\left(H_{n}-\sum_{j=1}^{n} \frac{1}{j 2^{j}}\right)+2^{n-1} \sum_{j=1}^{n} \frac{1}{2^{j}}$,

but $\sum_{j=1}^{n} \frac{1}{2^{j}}=1-\frac{1}{2^{n}}$, then (9) implies (3), that is [5]:

$\sum_{k=1}^{n} k\left(\begin{array}{l}n \\ k\end{array}\right) H_{k}=2^{n-1}-\frac{1}{2}+n \sum_{j=1}^{n}(-1)^{j+1}\left(\begin{array}{l}n \\ j\end{array}\right) \frac{2^{n-1-j}}{j}$.

From (9) with $\lambda=-\mu=1$ is immediate the identity:

$$
\sum_{k=1}^{n}(-1)^{k+1} k\left(\begin{array}{l}
n \\
k
\end{array}\right) H_{k}= \begin{cases}1, & n=1, \\
\frac{1}{1-n}, & n \geq 2 .\end{cases}
$$

\section{Conclusion}

The approach in this paper shows that the identities (2) and (3) obtained by PauleSchneider [8] can be deduced without the Mathematica package Sigma and the Zeilberger algorithm [9, 13].

\section{References}

[1] López-Bonilla J and López-Vázquez R (2017), Harmonic numbers in terms of Stirling numbers of the second kind, Prespacetime Journal, 8(2) : 233-234.

[2] Boyadzhiev KN (2009), Harmonic number identities via Euler's transform, J. Integer Sequences, 12 : Article 09.6.1 
[3] Carvajal-Gámez BE, López-Bonilla J and López-Vázquez R (2017), On harmonic numbers, Prespacetime Journal, 8(4) : 484-489.

[4] Gould HW (1972), combinatorial identities, Morgantown, W. Va.

[5] Guerrero-Moreno I, López-Bonilla J and López-Vázquez R (2017), Paule-Schneider's identities for harmonic numbers, Prespacetime Journal, 8(12): 1373-1376 to appear.

[6] Jin HT and Du DK (2015), Abel's lemma and identities on harmonic numbers, Integers, 15 : 211.

[7] Larcombe PG, Larseen ME and Fennessey EJ (2005), On two classes of identities involving harmonic numbers, Utilitas Math., 67 : 65-80.

[8] Paule P and Schneider C (2003), Computer proofs of a new family of harmonic number identities, Adv. Appl. Math. 31 : 359-378.

[9] Petkovsek M, Wilf HS and Zeilberger D (1996), $A=B$, symbolic summation algorithms, A. K. Peters, Wellesley, MA.

[10] Riordan J (1968), combinatorial identities, John Wiley \& Sons, New York.

[11] Schneider C (2000), An implementation of Karr's summation algorithm in Mathematica, Sém. Lothar. Combin. S43 b : 1-10.

[12] Spiess J (1990), Some identities involving harmonic numbers, Maths. of Comput. 55(192) : 839-863.

[13] Zeilberger D (1990), A fast algorithm for proving terminating hyper-geometric identities, Discrete Mathematics, 80(2) : 207-211. 